\title{
Searches for excited fermions at HERA
}

\section{Z. Zhang ${ }^{* \dagger}$}

Laboratoire de l'Accélérateur Linéaire, Univ. Paris-Sud et IN2P3/CNRS, Orsay, France

E-mail: zhangzq@lal.in2p3.fr

Final results from $\mathrm{H} 1$ are reported on searches for excited neutrinos $\left(v^{*}\right)$, electrons $\left(e^{*}\right)$ and quarks $\left(q^{*}\right)$ using the full data sample from the HERA ep collider corresponding to an integrated luminosity of up to $475 \mathrm{pb}^{-1}$ at a centre-of-mass energy of $320 \mathrm{GeV}$. The searches cover all electroweak decays of the excited fermions with subsequent hadronic or leptonic decays of the $W$ and $Z$ bosons. No evidence for first generation excited fermion production is found. Mass dependent exclusion limits on the production cross sections and on the ratio $f / \Lambda$ of the coupling to the compositeness scale are derived within gauge mediated models. A limit on $f / \Lambda$, independent of the relative couplings to the $\mathrm{SU}(2)$ and $\mathrm{U}(1)$ gauge boson, is also determined for the $v^{*}$ search. These final limits extend the excluded region to higher masses than has been possible in previous searches. The $e^{*}$ production via contact interactions is also addressed for the first time in $e p$ collisions.

European Physical Society Europhysics Conference on High Energy Physics, EPS-HEP 2009, July 16 - 222009

Krakow, Poland

\footnotetext{
* Speaker.

${ }^{\dagger}$ For the H1 Collaboration
} 
If leptons and quarks are composite particles, a natural consequence is the existence of excited leptons and quarks $\left(v^{*}, e^{*}\right.$ and $\left.q^{*}\right)$. HERA, the first electron ${ }^{1}$-proton collider built, provides an excellent environment for searching for singly produced excited fermions with mass values up to the maximum centre-of-mass energy of $320 \mathrm{GeV}$. The production of excited fermions at HERA proceeds through the exchange of a $W$ boson for $v^{*}$ and a $\gamma$ or $Z$ boson for $e^{*}$ and $q^{*}$ in the $t$ channel. Searches for these excited fermions have been actively performed at HERA since the very beginning starting from a first dataset of about $25 \mathrm{nb}^{-1}$ taken in 1992 until recently, with in total more than 10 publications from both $\mathrm{H} 1$ and ZEUS experiments [1, 2, 3]. This talk reports the final results obtained by H1 [3] using the full HERA data, which comprise $184 \mathrm{pb}^{-1}$ recorded in $e^{-} p$ collisions and $291 \mathrm{pb}^{-1}$ in $e^{+} p$ collisions. This represents more than a ten-fold, four-fold and twelve-fold increase in statistics for the $v^{*}, e^{*}$ and $q^{*}$ searches, respectively.

Although the searches would uncover any resonant state of appropriate coupling, independent of its spin and other dynamic properties, we have considered a specific phenomenological model [4] in which excited fermions are assumed to have spin $1 / 2$ and isospin $1 / 2$, sharing the other fermionic quantum number of the existing fermions. The left-handed and right-handed components of the excited fermions form weak iso-doublets $F_{L}^{*}$ and $F_{R}^{*}$. In order to prevent the light leptons from radiatively acquiring a large anomalous magnetic moment, only the right-handed component of the excited fermions is allowed to couple to light fermions. The interaction between excited fermions, gauge bosons and ordinary fermions is then described by the effective Lagrangian:

$$
\mathscr{L}_{\text {int. }}=\frac{1}{2 \Lambda} \bar{F}_{R}^{*} \sigma^{\mu v}\left[g f \frac{\tau^{a}}{2} W_{\mu \nu}^{a}+g^{\prime} f^{\prime} \frac{Y}{2} B_{\mu v}+g_{s} f_{s} \frac{\lambda^{a}}{2} G_{\mu \nu}^{a}\right] F_{L}+\text { h.c. . }
$$

The matrix $\sigma^{\mu v}$ is the covariant bilinear tensor, $\tau^{a}\left(\lambda^{a}\right)$ is the Pauli (Gell-Mann) matrix, $Y$ is the weak hypercharge, $W_{\mu \nu}^{a}, B_{\mu \nu}$ and $G_{\mu \nu}^{a}$ are the gauge fields associated with the $\mathrm{SU}(2), \mathrm{U}(1)$ and $\mathrm{SU}(3)_{\mathrm{C}}$ groups, respectively, and $g, g^{\prime}$ and $g_{s}$ are the corresponding standard electroweak and strong gauge couplings. The compositeness scale is set by the parameter $\Lambda$ which has units of energy, and $f, f^{\prime}$ and $f_{s}$ scale the $\mathrm{SU}(2), \mathrm{U}(1)$ and $\mathrm{SU}(3)_{\mathrm{C}}$ couplings, respectively. They can be interpreted as parameters setting different scales $\Lambda_{i}=\Lambda / f_{i}$ for the different gauge groups, thus allowing the composite fermions to have arbitrary coupling strengths with the three gauge bosons.

Whereas the production cross sections for $e^{*}$ and $q^{*}$ are comparable in $e^{-} p$ and $e^{+} p$ collisions, the $v^{*}$ production cross section in $e^{+} p$ is predicted to be much smaller than that in $e^{-} p$ by about two orders of magnitude for a $200 \mathrm{GeV} v^{*}$, due to the helicity dependence of the weak interaction and given the valence quark composition and density distribution the proton. Therefore the search for $v^{*}$ is only limited to the $e^{-} p$ data sample of $184 \mathrm{pb}^{-1}$.

The excited lepton (quark) may decay into a lepton (quark) and a gauge boson ( $\gamma, W$ and $Z$ ) with subsequent hadronic and leptonic decays of the $W$ and $Z$ bosons. For a given excited fermion mass value and assuming a numerical relation ${ }^{2}$ between $f$ and $f^{\prime}$, the excited fermion branching

\footnotetext{
${ }^{1}$ The term "electron" refers to both electron and positron, if not otherwise stated.

${ }^{2}$ For the excited quarks, one needs to fix $f_{s}$ as well, which is however assumed to be zero in the main analysis. This assumption leads to results which are complementary to the $q^{*}$ searches performed at the Tevatron, since at a $p \bar{p}$ collider excited quarks are dominantly produced in a quark-gluon fusion mechanism, which requires $f_{s} \neq 0$. The effect of non-zero values of $f_{s}$ is nevertheless studied for completeness.
} 


\begin{tabular}{|c|c|c|c|c|c|c|c|c|}
\hline Channel & Data & SM & Channel & Data & SM & Channel & Data & $\mathrm{SM}$ \\
\hline \multicolumn{3}{|c|}{$v^{*}$} & \multicolumn{3}{|c|}{$e^{*}$} & \multicolumn{3}{|c|}{$q^{*}$} \\
\hline \multirow{2}{*}{$v \gamma$} & \multirow{2}{*}{7} & \multirow{2}{*}{$12.3(3.0)$} & $e \gamma_{\text {ela. }}$ & 42 & $48(4)$ & \multirow{2}{*}{$q \gamma$} & \multirow{2}{*}{44} & \multirow{2}{*}{$46(8)$} \\
\hline & & & $e \gamma_{\text {inel. }}$ & 65 & $65(8)$ & & & \\
\hline$e W_{\rightarrow q \bar{q}}$ & 220 & $223(47)$ & $v W_{\rightarrow q \bar{q}}$ & 129 & $133(32)$ & $q W / Z_{\rightarrow q \bar{q}}$ & 341 & $326(78)$ \\
\hline$e W_{\rightarrow v \mu}$ & 0 & $0.40(0.05)$ & $\begin{array}{l}v W_{\rightarrow e v} \\
e Z_{\rightarrow v v}\end{array}$ & 4 & $4.5(0.7)$ & $q W_{\rightarrow e v}$ & 6 & $6.0(0.8)$ \\
\hline$e W_{\rightarrow v e}$ & 0 & $0.7(0.1)$ & $e Z_{\rightarrow q \bar{q}}$ & 286 & $277(62)$ & $q W_{\rightarrow \mu v}$ & 5 & $4.4(0.7)$ \\
\hline$v Z_{\rightarrow q \bar{q}}$ & 89 & $95(21)$ & $e Z_{\rightarrow e e}$ & 0 & $0.72(0.06)$ & $q Z_{\rightarrow e e}$ & 0 & $0.44(0.07)$ \\
\hline$v Z_{\rightarrow e e}$ & 0 & $0.19(0.05)$ & $e Z_{\rightarrow \mu \mu}$ & 0 & $0.52(0.05)$ & $q Z_{\rightarrow \mu \mu}$ & 0 & $0.87(0.11)$ \\
\hline
\end{tabular}

Table 1: Observed (Data) and predicted (SM) event yields for the studied excited fermion decay channels. The errors on the SM predictions given in parentheses include model and experimental systematic errors added in quadrature.

ratios are fixed and the production cross section depends only on $f / \Lambda$. The excited leptons $v^{*}$ and $e^{*}$ are expected not to have strong interactions and therefore this search is insensitive to $f_{s}$.

Two complementary coupling assignments $f=+f^{\prime}$ and $f=-f^{\prime}$ are studied. For $f=+f^{\prime}$, the excited neutrino has no tree-level electromagnetic coupling and therefore the photonic decay of the $v^{*}$ is forbidden whereas for $f=-f^{\prime}$, decays into $v \gamma, v Z$ and $v W$ are allowed. In addition, arbitrary ratios of $f^{\prime} / f$ in the range -5 to +5 are considered in the search for $v^{*}$. Similarly, in the case $f=-f^{\prime}$, the $e^{*}$ production cross section at HERA is small since the excited electron does not couple to the photon. Therefore, only the case $f=+f^{\prime}$ is considered in the analysis.

Under these assumptions, all dominant decay channels are analysed and summarised in Table 1. The total fraction of the decays channels covered is above $\sim 80 \%$ and up to $\sim 92 \%$ for the $f=-f^{\prime} v^{*}$ searches. Depending on the decay mode, the experimental signature of the signals can have isolated lepton(s)/photon, missing energy or jets in the final state. The dominant Standard Model (SM) processes are QED Compton scattering, neutral current and charged current deep-inelastic scattering and to a lesser extent photoproduction, lepton pair production and real $W$ boson production. The signal selection efficiency varies with decay channels and excited fermion masses and is in general higher for $v^{*}$ and $e^{*}$ searches and lower for $q^{*}$ searches with typically values around $30-50 \%$.

The event yields observed in all decay channels are in agreement with the corresponding SM expectations. Since no evidence for the production of excited fermions is observed, upper limits on the excited fermion cross sections and on the couplings $f / \Lambda$ are derived as a function of the reconstructed invariant mass of the excited fermions. Limits are presented in Fig. 1 at the $95 \%$ confidence level (CL) and are obtained from the mass spectra combining all decay channels using a modified frequentist approach which takes statistical and systematic uncertainties into account [5]. Also shown in Fig. 1 are limits obtained by the LEP and Tevatron experiments [6].

Within gauge mediated models and assuming $f / \Lambda=1 / M_{f^{*}}$, excited neutrinos, electrons and quarks with a mass lower than $213 \mathrm{GeV}, 272 \mathrm{GeV}$ and $252 \mathrm{GeV}$ are excluded at $95 \%$ confidence level, respectively. These results extend previously excluded domains at HERA and LEP and are complementary to $q^{*}$ searches performed at the Tevatron. 

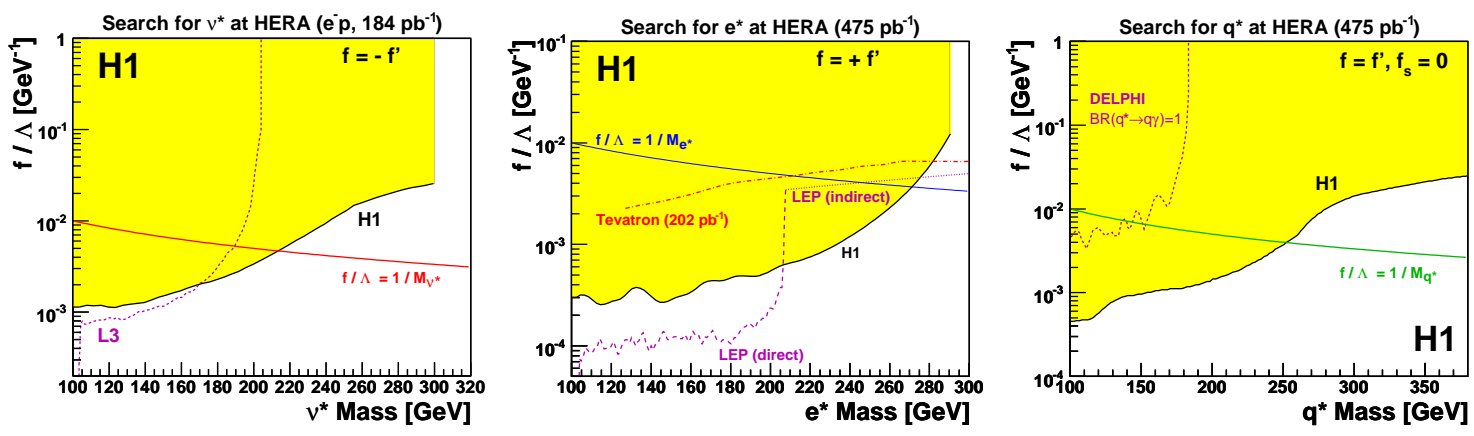

Figure 1: Exclusion limits at $95 \% \mathrm{CL}$ on the coupling $f / \Lambda$ as a function of the mass of the excited fermions with the assumptions (left) $v^{*}: f=-f^{\prime}$, (middle) $e^{*}: f=+f^{\prime}$ and (right) $q^{*}: f=f^{\prime}$ and $f_{s}$. The excluded domains based on the $\mathrm{H} 1$ data are represented by the shaded area. Values of the couplings above the curves are excluded. The dashed curves correspond to the exclusion limits obtained from LEP and Tevatron experiments.

For the $e^{*}$ search, gauge and four-fermion interactions are also considered together for $e^{*}$ production and decays for the first time in $e p$ collisions. In this scenario and assuming the same $\Lambda$ parameter in contact and gauge interactions, the limit on $1 / \Lambda$ improves only slightly, implying that the gauge interaction mechanism is dominant for the excited electron processes at HERA. A limit on $\Lambda$ as a function of the $e^{*}$ mass is also obtained at the Tevatron by considering single $e^{*}$ production via contact interactions only, followed by its gauge decay into an electron and a photon [7].

\section{References}

[1] H1 Collab., I. Abt et al., Nucl. Phys. B 396 (1993) 3; S. Aid et al, Nucl. Phys. B 483 (1997) 44; C. Adloff et al., Eur. Phys. J. C 17 (2000) 567; C. Adloff et al., Phys. Lett. B 525 (2002) 9; C. Adloff et al., Phys. Lett. B 548 (2002) 35.

[2] ZEUS Collab., M. Derrick et al., Phys. Lett. B 316 (1993) 207; M. Derrick et al., Z. Phys. C 65 (1995) 627; J. Breitweg et al., Z. Phys. C 76 (1997) 631; S. Chekanov et al., Phys. Lett. B 549 (2002) 32.

[3] H1 Collab., F.D. Aaron et al., Phys. Lett. B 663 (2008) 382; F.D. Aaron et al., Phys. Lett. B 666 (2008) 131; F.D. Aaron et al., Phys. Lett. B 678 (2009) 335.

[4] K. Hagiwara, S. Komamiya and D. Zeppenfeld, Z. Phys. C 29 (1985) 115; U. Baur, M. Spira and P.M. Zerwas, Phys. Rev. D 42 (1990) 815; F. Boudjema, A. Djouadi and J.L. Kneur, Z. Phys. C 57 (1993) 425.

[5] T. Junk, Nucl. Instrum. Meth. A 434 (1999) 435 [hep-ex/9902006].

[6] L3 Collab., P. Achard et al., Phys. Lett. B 568 (2003) 23 [hep-ex/0306016]; OPAL Collab., G. Abbiendi et al., Phys. Lett. B 544 (2002) 57 [hep-ex/0206061]; DELPHI Collab., J. Abdallah et al., Eur. Phys. J. C 37 (2004) 405 [hep-ex/0409058]; CDF Collab., D. Acosta et al., Phys. Rev. Lett. 94 (2005) 101802 [hep-ex/0410013]; DELPHI Collab., P. Abreu et al., Eur. Phys. J. C 8 (1999) 41 [hep-ex/9811005].

[7] D0 Collab., V.M. Abazov et al., Phys. Rev. D 77 (2008) 091102 [arXiv:0801.0877(hep-ex)]. 